INPLASY

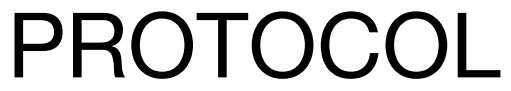

To cite: Qain et al. Platinum vs immunotherapy for unresectable esophageal cancer: a systematic review and meta-analysis protocol. Inplasy protocol 2020110012. doi:

10.37766/inplasy2020.11.0012

Received: 04 November 2020

Published: 04 November 2020

Corresponding author: Jiekun Qain

Qainjiekun111@163.com

Author Affiliation:

Fujian Medical University

Union Hospital

Support: Fujian Medical

University.

Review Stage at time of this submission: The review has not yet started.

Conflicts of interest: The authors have no conflicts of interest to disclose.

\section{Platinum vs immunotherapy for unresectable esophageal cancer: a systematic review and meta-analysis protocol}

\author{
Qain, J1; Tong, Z2; Zhang, Y3; Chen, C4.
}

Review question / Objective: Esophageal cancer is one of the most common malignant tumors, with early metastasis, highly malignant characteristics.Morbidity ranks 7th among all malignant tumors, and mortality ranks 6th. Esophageal adjuvant therapy can significantly improve overall survival in unresectable esophageal cancer patients. With the breakthrough and progress of immunotherapy, the possibility of curing esophageal cancer has greatly increased. Some clinical trials have reported that compared with traditional platinum-based chemotherapy, the use of programmed death 1 and programmed death ligand 1 inhibitors alone can benefit patients and effectively prolong their overall survival. We compare the efficacy of single immunotherapy with traditional platinum-based chemotherapy in a systematic review and meta-analysis to provide a reliable basis for clinicians.

INPLASY registration number: This protocol was registered with the International Platform of Registered Systematic Review and Meta-Analysis Protocols (INPLASY) on 04 November 2020 and was last updated on 04 November 2020 (registration number INPLASY2020110012).

\section{INTRODUCTION}

Review question / Objective: Esophageal cancer is one of the most common malignant tumors, with early metastasis, highly malignant characteristics.Morbidity ranks 7th among all malignant tumors, and mortality ranks 6th. Esophageal adjuvant therapy can significantly improve overall survival in unresectable esophageal cancer patients. With the breakthrough and progress of immunotherapy, the possibility 
of curing esophageal cancer has greatly increased. Some clinical trials have reported that compared with traditional platinum-based chemotherapy, the use of programmed death 1 and programmed death ligand 1 inhibitors alone can benefit patients and effectively prolong their overall survival. We compare the efficacy of single immunotherapy with traditional platinum-based chemotherapy in a systematic review and meta-analysis to provide a reliable basis for clinicians.

Condition being studied: We will evaluate the efficacy of postoperative adjuvant therapy (platinum based chemotherapy and immunotherapy) with or without radiotherapy for patients with unresectable esophageal cancer.

\section{METHODS}

Search strategy: We will search Pubmed, Medline, Embase, Web of Science, Cancerlit, Google Scholar, and the Cochrane Central Register of Controlled Trials for related studies published before December 1, 2019 without language restrictions.

Participant or population: The participants will be adults diagnosed with unresectable esophageal cancer histologically or cytologically confirmed who were treated with platinum-based chemotherapy, or immunotherapy.

Intervention: According to the means of postoperative chemotherapy for patients with unresectable esophageal cancer, the trials included will be divided into the following categories.

Comparator: The efficacy and safety of postoperative platinum-base chemotherapy versus immunotherapy for patients with unresectable esophageal cancer.

Study designs to be included: Randomized controlled trials (RCTs), quasi-RCTs, propensity score matched comparative studies and prospective cohort studies of interest, published 0 .
Eligibility criteria: Randomised controlled trials (RCTs) and quasi-RCTs published or unpublished will be included, which have been completed and compared postoperative platinum-base chemotherapy versus immunotherapy for patients with unresectable esophageal cancer.

Information sources: Jiekun Qian and Zhangwei Tong will search Pubmed (Medline), Embase, Google Scholar, Cancerlit, and the Cochrane Central Register of Controlled Trials for related studies published before June 20, 2021 without language restrictions.

Main outcome(s): The primary outcomes will be postoperative overall survival of patients with unresectable esophageal cancer who were treated with chemotherapy.

Additional outcome(s): We will assess the 5-year survival, median survival, recurrence-free survival, quality of life, and adverse events or complications of patients with unresectable esophageal cancer who were treated with chemotherapy.

Data management: The two authors (JKQ, ZWT) will extract the following data independently from the studies included. • Study characteristics and methodology: publication date, the first author, country, randomization, study design, periods of data collection, follow-up duration, total duration of study, and withdrawals, et al. • Participant characteristics: gender, age, tumor stage, pathology diagnosis, ethnicity, performance status, history of smoking, pathologic tumor size, and inclusion criteria, et al. - Interventions: therapeutic means, drugs, dosage, modality and frequency of administration, et al. • Outcome and other data: overall survival, 5-year survival, median survival, diseasefree survival, $95 \%$ confidence intervals, recurrence time, quality of life, adverse events, and complications, et al. We will record all the date extracted in a predesigned table and consult the first author of the trial by e-mail before determining 
eligibility, if the reported data of which are unclear or missing.

Quality assessment / Risk of bias analysis: Two authors (JKQ, ZWT) will use the Cochrane Handbook for Systematic Reviews of Interventions to assess the risk of bias of each study included independently based on the following ranges: random sequence generation (selection bias); allocation concealment (selection bias); blinding of participants and personnel (performance bias); blinding of outcome assessment (detection bias); incomplete outcome data (attrition bias); selective outcome reporting(reporting bias); other bias[22]. Each domain will be assessed as high, low or uncertain risk of bias. The results and details of assessment will be reported on the risk of bias graph.

Strategy of data synthesis: The data will be synthesised by Review Manager 5.3 software. We will conduct a systematic review and meta-analysis only if the data gathered from included trials are judged to be similar enough to ensure a result that is meaningful. The Chi2 test and 12 statistic will be used to assess statistical heterogeneity among the trials included in matched pairs comparison for standard meta-analysis. The random effect model will be applied to analyse the data, if there is substantial heterogeneity (p50\%) and the trials will be regarded to be obvious heterogeneous. Otherwise, we will utilize fixed effect model to analyse the data. Mantel-Haenszel method will be adopted to pool of the binary data. The results will be reported in the form of relative risk (RR) between $95 \%$ confidence interval $(\mathrm{Cl})$ of the date. The continuous data will be pooled by inverse variance analysis method and the results will be shown in the form of standardized mean difference (SMD) with 95\% confidence interval $(\mathrm{Cl})$ of the date.

Subgroup analysis: If there is high heterogeneity (12 statistic $>50 \%)$ and the data are sufficient, subgroup analysis will be conducted to search potential causes of heterogeneity. Subgroup analysis will be performed in different methods of postoperative adjuvant therapy, ethnicity, history of smoking, tumor stage, and type of operation.

Sensibility analysis: Sensitivity analysis will be conducted to assess the reliability and robustness of the aggregation results via eliminating trials with high bias risk.

Language: Without any language restrictions.

Country(ies) involved: China.

Keywords: Esophageal cancer, immunotherapy, platinum-based chemotherapy.

Contributions of each author:

Author 1 - Jiekun Qain - Drafted the manuscript.

Author 2 - Zhangwei Tong - Provided statistical expertise.

Author 3 - Yannan Zhang - Contributed to the development of the selection criteria, and the risk of bias assessment strategy.

Author 4 - Chun Chen - provided feedback and approved the final manuscript. 